\title{
GT Regimen
}

National Cancer Institute

\section{Source}

National Cancer Institute. GT Regimen. NCI Thesaurus. Code C63422.

A chemotherapy regimen consisting of paclitaxel and gemcitabine used as a treatment for recurrent and metastatic breast cancer. 